\title{
Soluble CD40 Levels in Plasma Are Associated with Cardiovascular Disease and in Carotid Plaques with a Vulnerable Phenotype
}

\author{
Annelie Shami, ${ }^{a}$ Andreas Edsfeldt,, ${ }^{\mathrm{a}, \mathrm{b}}$ Eva Bengtsson, ${ }^{\mathrm{a}}$ Jan Nilsson, ${ }^{\mathrm{a}}$ Angela C. Shore, ${ }^{\mathrm{c}}$ Andrea Natali, ${ }^{\mathrm{d}}$ \\ Faisel Khan, ${ }^{\mathrm{e}}$ Esther Lutgens, ${ }^{\mathrm{f}, \mathrm{g}, \mathrm{h}}$ Isabel Gonçalves ${ }^{\mathrm{a}, \mathrm{b}}$ \\ ${ }^{a}$ Department of Clinical Sciences Malmo, Clinical Research Center, Lund University, Malmo, Sweden \\ ${ }^{b}$ Department of Cardiology, Skane University Hospital, Lund University, Malmo, Sweden \\ ' Diabetes and Vascular Medicine, University of Exeter Medical School, National Institute for Health Research Exeter Clinical Research Facility, \\ Exeter, UK \\ ${ }^{\mathrm{d}}$ Department of Clinical and Experimental Medicine, University of Pisa, Pisa, Italy \\ ${ }^{\text {e }}$ Division of Molecular and Clinical Medicine, University of Dundee, Dundee, UK \\ ${ }^{f}$ Department of Medical Biochemistry, Amsterdam Cardiovascular Sciences, Amsterdam University Medical Centers, University of Amsterdam, \\ Amsterdam, The Netherlands \\ ${ }^{9}$ Institute for Cardiovascular Prevention (IPEK), Ludwig Maximilian University of Munich, Munich, Germany \\ ${ }^{\mathrm{h}}$ German Center for Cardiovascular Research (DZHK), Partner Site Munich Heart Alliance, Munich, Germany
}

Background and Purpose CD40 and CD40 ligand (CD40L) are costimulatory molecules of the tumor necrosis factor receptor superfamily and well known for their involvement in inflammatory diseases: atherosclerotic mouse models with disrupted CD40 signalling develop lesions of reduced size with a more stable plaque profile. This study investigated the potential of plasma and intraplaque levels of CD40 and CD40L as markers for cardiovascular disease (CVD) in humans and their association with plaque stability.

Methods Soluble CD40 and CD40L (SCD40L) were measured in plasma in 1,437 subjects from The SUrrogate markers for Micro- and Macro-vascular hard endpoints for Innovative diabetes Tools (SUMMIT) cohort. Intra-plaque levels of sCD40 and sCD40L were measured in atherosclerotic plaque homogenates from 199 subjects of the Carotid Plaque Imaging Project (CPIP) cohort.

Results Both plasma sCD40 and SCD40L levels were elevated in individuals with prevalent stroke, while SCD40 levels also were higher in individuals with a prior acute myocardial infarction. Plasma levels of sCD40 correlated with carotid intima-media thickness and total carotid plaque area and were associated with risk of cardiovascular events over a 3-year follow-up period. Intra-plaque levels of SCD40 and SCD40L were associated with plaque components characteristic for plaque vulnerability and extracellular matrix remodelling.

Conclusions Higher plasma SCD40 and SCD40L levels are associated with prevalent CVD. Plasma sCD40 levels also correlate with the severity of carotid atherosclerosis and predict future cardiovascular events, while intra-plaque levels correlate with a vulnerable plaque phenotype. Our findings thus demonstrate that elevated levels of SCD40 and SCD40L are markers of CVD.
Correspondence: Annelie Shami Department of Clinical Sciences Malmo, Lund University, Jan Waldenströms gata 35, 21428 Malmo, Sweden Tel: +46-(0)40-391187 E-mail: annelie.shami@med.lu.se https://orcid.org/0000-0002-6976-4397

Received: January 11, 2021

Revised: April 30, 2021

Accepted: May 17, 2021

Keywords CD40; CD40 ligand; Atherosclerosis; Carotid arteries; Inflammation 


\section{Introduction}

Cardiovascular disease (CVD) is the leading cause of mortality worldwide, accounting for 17.9 million deaths each year. ${ }^{1}$ The main underlying cause is atherosclerosis. Compromised stability of atherosclerotic plaques results in thrombus formation, and, consequently, stroke or myocardial infarction (MI) depending on the affected territory. Characteristics of a classically rupture-prone or vulnerable plaque include a thin fibrous cap, abundance of lipids, a prominent necrotic core and infiltration by immune cells, such as macrophages and T cells, ${ }^{2}$ while eroded lesions are less inflamed and rich in proliferating smooth muscle cells (SMCs), proteoglycans and hyaluronan, and neovascularization. ${ }^{3}$ Ideally, ameliorating atherosclerosis involves two steps: (1) finding a reliable method to identify individuals with vulnerable plaques to start treatment in time to prevent ruptures-and subsequently symptoms, and (2) identifying key players driving destabilization in rupture-prone plaques. Addressing both steps, this study not only evaluates the CD40/CD40L signalling dyad as a potential CVD marker but also explores its biological association with a vulnerable plaque phenotype.

CD40 and CD40 ligand (CD40L) are costimulatory molecules and members of the tumor necrosis factor (receptor) superfamily (TNF(R)SF). ${ }^{4} \mathrm{CD} 40$ is expressed on a variety of immune and non-immune cells, including B cells, monocytes/macrophages, dendritic cells, SMCs and endothelial cells. CD40L is mainly expressed by activated T cells and platelets, but expression has also been described on B cells, as well as on endothelial, epithelial, and $\mathrm{SMCs}^{5} \mathrm{CD} 40$ signalling is propagated via adaptor proteins-the TNF receptor-associated factors ${ }^{6}$-and modulates a multitude of immunological responses. ${ }^{5}$ Atherosclerotic mouse models (apolipoprotein $\mathrm{E}\left[\mathrm{Apo}^{-1-}\right]$, low-density lipoprotein receptor $\left[\mathrm{LDLr}^{-1}\right]$ ) with disrupted $\mathrm{CD} 40$ signalling develop lesions of reduced size with a more stable plaque profile, an effect achieved by CD40/CD40L knock out models, as well as by small molecule inhibitors and antibodies inhibiting the $\mathrm{CD} 40$ signalling pathway. ${ }^{6-9}$

While not much is known about the specific actions of CD40 in human CVD, an association for CD40L in CVD has been suggested. Increased levels of platelet CD40L have been reported in patients with hypercholesterolemia ${ }^{10}$ and plasma levels of soluble CD40L (sCD40L) were increased in patients with acute coronary syndromes. ${ }^{11}$ Furthermore, clinical evidence for involvement of SCD40L in CVD include association with cardiovascular (CV) events in coronary artery disease, ${ }_{11}^{11}$ recurrent cardiac events and future events in healthy women, ${ }^{12}$ carotid intima-media thickness (IMT) and hypertension, ${ }^{13}$ and via correla- tions with other biomarkers of inflammation. ${ }^{14} \mathrm{~A}$ number of studies, however, have found no associations between plasma SCD40L and CVD ${ }^{15,16}$ or long-term CV (or all-cause) mortality. ${ }^{17}$ Further clarification is thus essential to determine the value of SCD40L as a marker of CVD, and to investigate whether SCD40 may be a useful complement or replacement biomarker.

To address the potential of SCD40 and SCD40L as markers of $C V D$, we have analysed plasma and carotid artery plaque levels of CD40/CD40L for associations with CV events, as well as carotid IMT and plaque vulnerability markers in two large human cohorts.

\section{Methods}

Extended methods are provided in the Supplementary Methods.

\section{Study cohorts and samples}

Plasma samples were included from 1,438 subjects (Figure 1) of the SUrrogate markers for Micro- and Macro-vascular hard endpoints for Innovative diabetes Tools (SUMMIT) study ${ }^{18}$ population, recruited between December 2010 and April 2013 from existing population cohorts and hospital registries at the university hospitals in Malmö (Sweden), Pisa (Italy), Dundee and Exeter (UK).

Carotid plaques ( $n=198)$ were collected from patients during carotid endarterectomies at the Vascular Department of Skane University Hospital (Malmö, Sweden) between 2005 and 2010 (The Carotid Plaque Imaging Project [CPIP]). ${ }^{19}$ Immediately during surgery, removed plaques were snap-frozen in liquid ni-

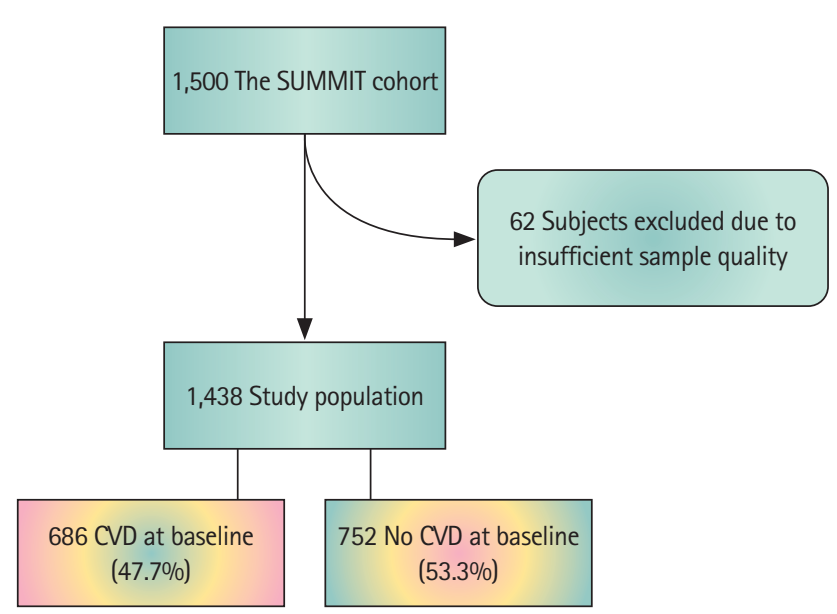

Figure 1. Flow chart showing the study population of the The SUrrogate markers for Micro- and Macro-vascular hard endpoints for Innovative diabetes Tools (SUMMIT) cohort, including excluded subjects and subjects with/without cardiovascular disease (CVD). 
trogen. Histological analyses were performed on portions (1 $\mathrm{mm}$ thick) cut from the most stenotic plaque region and embedded in optimal cutting medium (Sakura Finetek Europe BV, Tokyo, Japan). Plaque homogenates were prepared from the rest of the plaques and used for all other plaque component quantifications. Clinical characteristics of the included patients are summarized in Supplementary Table 1. The studies were approved by the local ethical review boards and were carried out in accordance with the principles of the Declaration of Helsinki. There was no overlap between subjects of the two study cohorts.

\section{Statistics}

Shapiro-Wilk and D'Agostino-Pearson omnibus K2 tests were used to assess Gaussian distribution. Variables found to be normally distributed are shown as mean with standard deviation while non-normally distributed variables are shown as median with interquartile range (IOR). For analysis of plasma, plaque histology sections and homogenates, the Mann-Whitney U test was used to compare groups and Spearman's rank correlation was used for continuous variables. For comparisons between categorical variables, the chi-square test was used. A logistic regression model was used to test for associations with $\mathrm{CV}$ events and mortality in the SUMMIT cohort. The model was adjusted for age, diabetes and prevalent ischemic stroke, acute myocardial infarction (AMI), and hypertension as covariates. A $P$-value of $<0.05$ was considered statistically significant. Statistical analysis was performed using SPSS version 26.0 (IBM Co., Armonk, NY, USA) and GraphPad Prism version 8.0.0 for Windows (GraphPad Software, San Diego, CA, USA, www. graphpad.com). Adjustments for multiple comparisons were done when appropriate using the Holm-Šídák test.

\section{Results}

\section{Plasma sCD40 levels are associated with stroke and acute myocardial infarction}

To assess associations between SCD40, sCD40L and CVD, we analysed the plasma from 1,438 subjects from the SUMMIT cohort using Proximity Extension Assay (PEA). Comparatively high $\mathrm{SCD} 40$ levels were associated with prevalent ischemic stroke $\left(P=3.0 \times 10^{-5}\right), \mathrm{MI}(P=0.016)$, and hypertension $(P=0.00021)$ (Table 1 and Supplementary Tables 2 and 3$)$. The association with stroke remained after adjusting for age, diabetes, hypertension, and $\mathrm{Ml}$ (odds ratio [OR], 1.5; 95\% confidence interval $[\mathrm{Cl}$, 1.084 to $1.959 ; P=0.013$ ) and the association with $\mathrm{Ml}$ after adjusting for age, diabetes, hypertension, and stroke $(\mathrm{OR}, 1.2 ; 95 \% \mathrm{Cl}, 1.006$ to $1.393 ; P=0.042)$.

Similar to SCD40, higher plasma SCD40L levels were associated with prevalent stroke $(P=0.020)$. However, SCD40L levels were not associated with hypertension or prevalent $\mathrm{AMI}$, and associations did not remain after adjusting for the aforementioned confounders (stroke: $O \mathrm{R}, 1.1 ; 95 \% \mathrm{Cl}, 0.976$ to 1.304 ; $P=0.103$; and $\mathrm{Ml}: \mathrm{OR}, 1.0 ; 95 \% \mathrm{Cl}, 0.968$ to $1.125 ; P=0.270$ ).

\section{Plasma sCD40 levels are associated with carotid plaque burden and arterial stiffness}

Plaque burden in the carotid artery was assessed via ultrasound imaging at study inclusion and we searched for possible associations between SCD40 and SCD40L and plaque burden. A positive Spearman's rank correlation was found between both plasma SCD40 and SCD40L levels and total plaque area ( $r=0.355, P<1 \times 10^{-16}$ and $r=0.409, P<1 \times 10^{-16}$, respectively) (Table 2). The association between SCD40 and SCD40L and carotid plaque burden was also reflected by positive correlations with

Table 1. Spearman correlations between the circulating levels of SCD40 and SCD40L (arbitrary unit, SUMMIT cohort) and carotid plaque burden

\begin{tabular}{|c|c|c|c|c|c|c|}
\hline \multirow[t]{2}{*}{ Vascular ultrasound* } & \multicolumn{3}{|c|}{$\begin{array}{c}\operatorname{CD} 40 \\
(n=1,438 \text { at inclusion; } n=798 \text { at follow-up) }\end{array}$} & \multicolumn{3}{|c|}{$\begin{array}{c}\text { CD40L } \\
(\mathrm{n}=1,438 \text { at inclusion; } \mathrm{n}=798 \text { at follow-up) }\end{array}$} \\
\hline & r & $P$ & $P$, adjusted ${ }^{+}$ & r & $P$ & $P$, adjusted \\
\hline 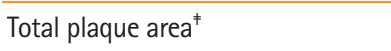 & 0.355 & $<1 \times 10^{-16}$ & $<1 \times 10^{-16}$ & 0.409 & $<1 \times 10^{-16}$ & $<1 \times 10^{-16}$ \\
\hline Maximum plaque area $\left(\mathrm{mm}^{2}\right)$ & 0.322 & $<1 \times 10^{-16}$ & $<1 \times 10^{-16}$ & 0.340 & $<1 \times 10^{-16}$ & $<1 \times 10^{-16}$ \\
\hline No. of plaques, right carotid artery & 0.354 & $<1 \times 10^{-16}$ & $<1 \times 10^{-16}$ & 0.152 & $2.0 \times 10^{-8}$ & $1.0 \times 10^{-7}$ \\
\hline No. of plaques, left carotid artery & 0.398 & $<1 \times 10^{-16}$ & $<1 \times 10^{-16}$ & 0.142 & $1.9 \times 10^{-7}$ & $7.6 \times 10^{-7}$ \\
\hline Plaque length (mm) & 0.316 & $<1 \times 10^{-16}$ & $<1 \times 10^{-16}$ & 0.286 & $1.3 \times 10^{-15}$ & $9.3 \times 10^{-15}$ \\
\hline Plaque height (mm) & 0.223 & $5.9 \times 10^{-10}$ & $1.2 \times 10^{-9}$ & 0.164 & 0.000006 & 0.000018 \\
\hline Lumen diameter reduction (\%) & 0.129 & 0.00041 & 0.00041 & 0.224 & $5.2 \times 10^{-10}$ & $3.1 \times 10^{-9}$ \\
\hline Pulse wave velocity & 0.247 & $8.0 \times 10^{-8}$ & $<1 \times 10^{-16}$ & 0.057 & 0.052 & 0.052 \\
\hline
\end{tabular}

sCD40, soluble CD40; sCD40L, soluble CD40 ligand; SUMMIT, The SUrrogate markers for Micro- and Macro-vascular hard endpoints for Innovative diabetes Tools.

${ }^{*}$ Measured at study inclusion; ${ }^{+} P$-value adjusted for multiple comparisons; ${ }^{~}$ Sum of all plaque areas/patient. 
Table 2. Spearman correlations between the circulating levels of sCD40 and SCD40L (arbitrary unit, SUMMIT cohort) and IMT in the CCA and the carotid bulb region assessed by vascular ultrasound (measured at study inclusion)

\begin{tabular}{|c|c|c|c|c|c|c|}
\hline \multirow[t]{2}{*}{ IMT measurements } & \multicolumn{3}{|c|}{$\begin{array}{c}\text { CD40 } \\
\text { ( } n=1,438 \text { at inclusion; } n=798 \text { at follow-up) }\end{array}$} & \multicolumn{3}{|c|}{$\begin{array}{c}\text { CD40L } \\
(n=1,438 \text { at inclusion; } n=798 \text { at follow-up) }\end{array}$} \\
\hline & $r$ & $P$ & $P$, adjusted* & $r$ & $P$ & $P$, adjusted \\
\hline \multicolumn{7}{|l|}{ Right } \\
\hline Mean IMT in CCA & 0.139 & $2.7 \times 10^{-7}$ & $1.1 \times 10^{-6}$ & 0.053 & 0.048 & 0.256 \\
\hline Max IMT in CCA & 0.122 & 0.000006 & 0.000018 & 0.012 & 0.65 & 0.903 \\
\hline Mean IMT in bulb & 0.234 & $1.4 \times 10^{-16}$ & $6.6 \times 10^{-16}$ & 0.081 & 0.005 & 0.039 \\
\hline Max IMT in bulb & 0.268 & $<1 \times 10^{-16}$ & $<1 \times 10^{-16}$ & 0.077 & 0.008 & 0.055 \\
\hline \multicolumn{7}{|l|}{ Left } \\
\hline Mean IMT in CCA & 0.088 & 0.001 & 0.0020 & 0.042 & 0.12 & 0.472 \\
\hline Max IMT in CCA & 0.073 & 0.007 & 0.0070 & 0.000 & 0.99 & 0.990 \\
\hline Mean IMT in bulb & 0.203 & $9.1 \times 10^{-13}$ & $4.5 \times 10^{-12}$ & 0.017 & 0.54 & 0.903 \\
\hline Max IMT in bulb & 0.240 & $<1 \times 10^{-16}$ & $<1 \times 10^{-16}$ & 0.038 & 0.19 & 0.570 \\
\hline
\end{tabular}

sCD40, soluble CD40; sCD40L, soluble CD40 ligand; SUMMIT, The SUrrogate markers for Micro- and Macro-vascular hard endpoints for Innovative diabetes Tools; IMT, intima-media thickness; CCA, common carotid artery.

${ }^{*} P$-value adjusted for multiple comparisons.

Table 3. Logistic regression analysis of association between the circulating levels of sCD40 (sCD40, SUMMIT cohort) and cardiovascular events (adjusted for confounding factors*) $(n=1,438)$

\begin{tabular}{lccc}
\hline Variable & Confidence interval & Odds ratio & $P$ \\
\hline sCD40 & $1.042-1.625$ & 1.3 & 0.020 \\
Age & $0.996-1.042$ & 1.0 & 0.109 \\
Diabetes & $0.759-1.681$ & 1.1 & 0.549 \\
Hypertension & $0.596-1.319$ & 0.9 & 0.553 \\
Acute myocardial infarction & $1.178-2.467$ & 1.7 & 0.005 \\
Stroke & $1.058-3.507$ & 1.9 & 0.032 \\
\hline
\end{tabular}

SCD40, soluble CD40; SUMMIT, The SUrrogate markers for Micro- and Macro-vascular hard endpoints for Innovative diabetes Tools.

${ }^{*}$ Odds ratios were adjusted for age (years) and prevalent diabetes, hypertension, prior acute myocardial infarction, and stroke (yes vs. no).

overall number of plaques, measurements of plaque length, height and area, as well as with lumen diameter reduction (Table 2).

Arterial stiffness-a well-known factor associated with atherosclerosis progression ${ }^{20}$-was studied by pulse wave velocity at study inclusion. Plasma levels of sCD40, but not sCD40L, correlated with carotid arterial stiffness $\left(r=0.247, P<1 \times 10^{-16}\right)$ (Table 2). Furthermore, sCD40 plasma levels measured at study inclusion showed a positive correlation with both mean and maximum IMT in the right and left common carotid artery (CCA), as well as in in the plaque bulb regions (Table 3). Association was also found between plasma sCD40L and mean IMT in the bulb region of the right carotid artery (Table 3 ).

\section{Plasma sCD40L levels are associated with carotid artery plaque progression and SCD40 levels with future cardiovascular events}

To elucidate whether plasma sCD40 and sCD40L levels predicted carotid atherosclerosis progress, in addition to correlat- ing with plaque burden at study inclusion, we compared the initial IMT measurements calculated via ultrasound imaging with IMT measurements taken after 3 years. Plasma sCD40L, but not sCD40, levels were associated with change-specifically plaque growth-in maximum IMT in the CCA and mean and maximum IMT in the bulb region of the plaques (Supplementary Table 4).

As SCD40L levels correlated with carotid plaque progression, we then investigated the potential for plasma sCD40 and sCD40L levels to predict future CV events using logistic regression. Interestingly, the level of sCD40, but not sCD40L, in plasma was associated with the occurrence of fatal or non-fatal $\mathrm{CV}$ events during the 3 years follow-up period (OR, 1.3; 95\% $\mathrm{Cl}, 1.042$ to 1.625$)$ after adjusting for age, diabetes, hypertension as well as prevalent $\mathrm{AMI}$ and stroke at baseline (Table 3). Neither sCD40 nor sCD40L was associated with mortality (CV or all-cause). Considering SCD40 (with confounders age, diabetes, hypertension as well as prevalent $\mathrm{MI}$ and stroke at baseline) in a multiple logistic regression, the area under the curve 

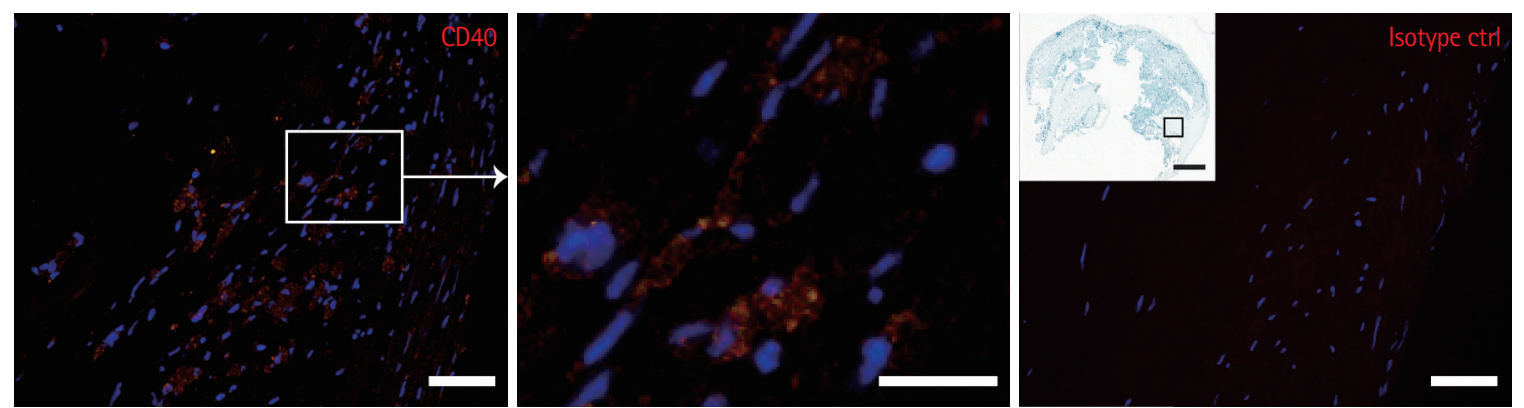

A
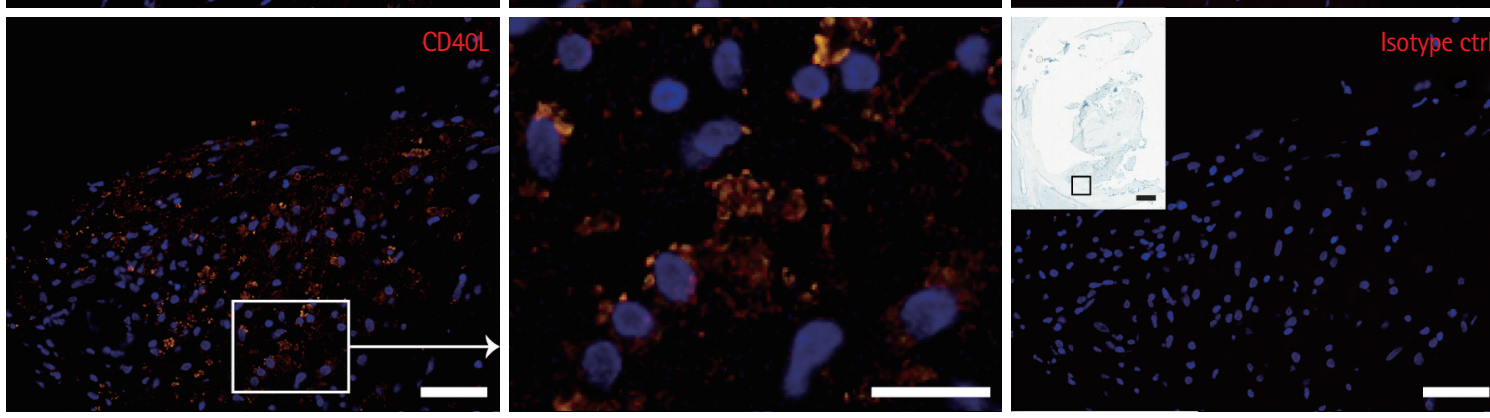

B
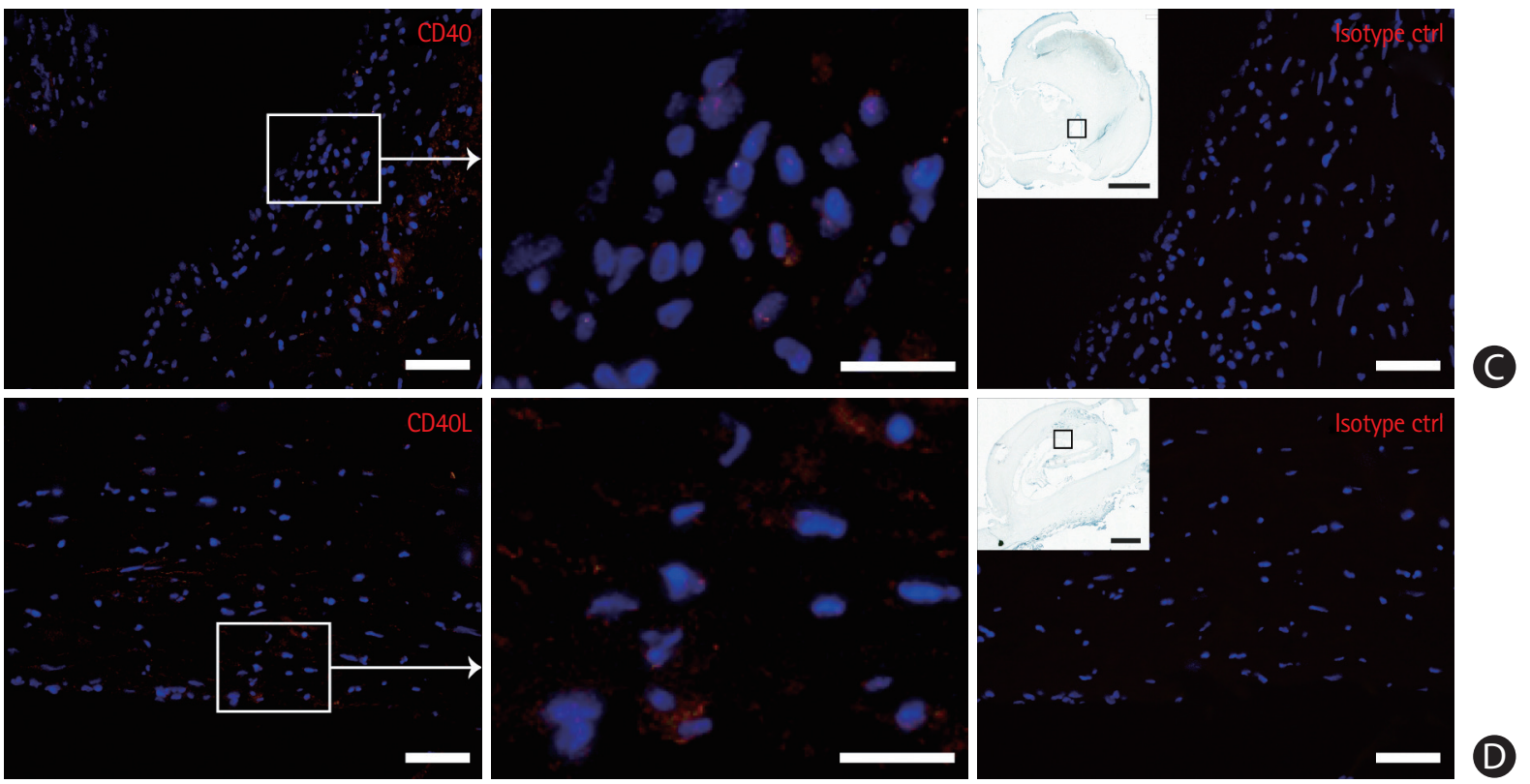

Figure 2. CD40 and CD40 ligand (CD40L) localization in endarterectomy plaques. CD40 and CD40L immunoreactivity was found in particular in the shoulder $(A, B)$ and cap $(C, D)$ regions. Squares in insets mark enlarged plaque regions. Scale bars are $50 \mu \mathrm{m}$ (far left and right image columns), $20 \mu \mathrm{m}$ (middle column), and $1 \mathrm{~mm}$ (insets, far left column) ( $\mathrm{n}=22$ plaques).

achieved was $0.629(95 \% \mathrm{Cl}, 0.582$ to 0.677$)$, with sensitivities and specificities for varying cut-offs of the predicted values (10\% to 90\% quantiles) presented in Supplementary Table 5.

\section{CD40 and CD40L have similar expression patterns} in human carotid plaques

To further explore the presence of CD40 and CD40L in atherosclerosis we examined their expression pattern in carotid endarterectomy plaques from the CPIP cohort (Figure 2). Immunofluorescence staining was performed on sections obtained from the most stenotic plaque region. CD40 and CD40L expression was observed in several plaque parts, in particular in the shoulder and cap regions. Immunoreactivity was also found-though far less prominent-in the base of the plaque.

\section{sCD40 is associated with a vulnerable plaque phenotype}

To further explore the presence of CD40 and CD40L in atherosclerosis we examined their expression in homogenates from 198 carotid endarterectomy plaques of the CPIP cohort (here 
Table 4. Spearman correlations of CD40 and CD40L (A.U., CPIP cohort) measured in plaque homogenate versus plaque elements

\begin{tabular}{|c|c|c|c|c|c|c|}
\hline \multirow{2}{*}{ Variable } & \multicolumn{3}{|c|}{ CD40 $(n=198)$} & \multicolumn{3}{|c|}{ CD40L $(n=198)$} \\
\hline & $r$ & $P$ & $P$, adjusted ${ }^{*}$ & $r$ & $P$ & $P$, adjusted \\
\hline \multicolumn{7}{|l|}{ Histology (area \%) } \\
\hline Smooth muscle a-actin & -0.070 & 0.33 & 0.33 & -0.142 & 0.048 & 0.094 \\
\hline Calcium (van Kossa) & -0.208 & 0.004 & 0.016 & -0.268 & 0.00017 & 0.00068 \\
\hline Oil Red 0 & 0.199 & 0.005 & 0.016 & 0.043 & 0.55 & 0.55 \\
\hline Vulnerability index ${ }^{+}$ & 0.178 & 0.014 & 0.029 & 0.180 & 0.014 & 0.041 \\
\hline \multicolumn{7}{|l|}{ Cytokines (pg/g) } \\
\hline CXCL1 & 0.228 & 0.002 & 0.012 & 0.268 & 0.002 & 0.008 \\
\hline IL-6 & 0.305 & 0.00032 & 0.0022 & 0.235 & 0.001 & 0.007 \\
\hline IL-12p70 & 0.211 & 0.004 & 0.020 & 0.230 & 0.002 & 0.008 \\
\hline Tumor necrosis factor a & 0.391 & $3.4 \times 10^{-8}$ & $3.1 \times 10^{-7}$ & 0.290 & $6.9 \times 10^{-5}$ & 0.0006 \\
\hline CCL5 & 0.021 & 0.772 & 0.772 & 0.200 & 0.007 & 0.014 \\
\hline CCL2 & 0.175 & 0.017 & 0.034 & 0.186 & 0.001 & 0.007 \\
\hline CCL4 & 0.202 & 0.006 & 0.020 & 0.186 & 0.011 & 0.014 \\
\hline PDGF-AA/AB/BB & 0.268 & 0.0002 & 0.0018 & 0.243 & 0.001 & 0.007 \\
\hline \multicolumn{7}{|l|}{ Plaque components $(\mathrm{mg} / \mathrm{g}$ ) } \\
\hline $\operatorname{oxLDL}(\mu \mathrm{U} / \mathrm{g})$ & 0.236 & 0.001 & 0.004 & 0.259 & 0.00041 & 0.0037 \\
\hline
\end{tabular}

CD40L, CD40 ligand; A.U., arbitrary unit; CPIP, Carotid Plaque Imaging Project; CXCL1, C-X-C motif chemokine ligand 1; IL, interleukin; CCL, chemokine (C-C motif) ligand; PDGF, platelet-derived growth factor; oxLDL, oxidized low density lipoprotein.

${ }^{*} P$-value adjusted for multiple comparisons; ${ }^{+}$Calculated as $\left(\mathrm{CD} 8^{+}+\right.$glycophorin $\mathrm{A}^{+}+0$ il Red $\mathrm{O}^{+}$area\%) / (smooth muscle a-actin ${ }^{+}+$collagen $^{+}+$von Kossa ${ }^{+}$ area\%).

comprised of both the soluble and cell associated isoforms). Disrupted CD40 signalling in a murine atherosclerosis model results in increased stabilisation characterised by reductions in lipid core size and inflammatory cell presence accompanied by increased collagen content. ${ }^{6-9}$ We therefore investigated whether intra-plaque levels of these molecules are similarly associated with a vulnerable plaque profile in human lesions. Indeed, we found SCD40 plaque levels measured in plaque homogenates of the CPIP cohort to correlate with plaque components collectively indicating a vulnerable plaque phenotype (Table 4). Accordingly, there was a correlation between sCD40 and lipid content (visualized on tissue sections by Oil Red $\mathrm{O}_{\text {, }}$ $r=0.199, P=0.012$; and oxidized low density lipoprotein [oxLDL] measured in plaque homogenate, $r=0.236, P=0.004)$, whereas a negative correlation was found with calcium (von Kossa, $r=$ $-0.208, P=0.012$ ). The level of $S C D 40 \mathrm{~L}$ in plaque showed an overall association with a vulnerable plaque phenotype, albeit weaker than that for SCD40 levels (Table 4). SCD40L levels correlated with the plaque content of lipids (oxLDL measured in plaque homogenate, $r=0.259, P=0.0037$ ), while there was an inverse correlation with plaque levels of calcium (von Kossa, $r=-0.268, P=0.00034)$. Finally, for further plaque profile evaluation a vulnerability index was calculated, which was also found to correlate with both $\operatorname{SCD} 40(\mathrm{r}=0.178, P=0.029)$ and sCD40L ( $r=0.180, P=0.041$ ) plaque levels.

CD40 ligation has been previously well-described to induce pro-inflammatory cytokine and chemokines, including interleukin (IL)-1 $\beta$, IL-6, IL-8, IL-12, TNF- $a$, and chemokine (C-C motif) ligand 5 (CCL5) in vitro. ${ }^{21-23}$ Concordantly, sCD40 levels correlated with both IL-12p70 and CCL2 measured in plaque homogenate, as well as with several other inflammatory cytokines and chemokines, including IL-6, TNF- $a$, CCL4, and C-X-C motif chemokine ligand 1 (CXCL1) (Table 4). Correlation was also found with platelet-derived growth factor (PDGF). Similarly, sCD40L levels correlated with the inflammatory cytokines and chemokines IL-12p70, CCL2, IL-6, TNF- $a$, CCL4, CCL5, CXCL1 as well as with PDGF (Table 4).

\section{sCD40 plaque levels are associated with features of extracellular matrix turnover}

The reported increase in fibrous cap thickness and collagen content in plaques from CD40-deficient mice has been proposed to result from the accompanying decreased plaque expression and activity of matrix metalloproteinase (MMP)-2 and -9 together with increased tissue inhibitor of metalloproteinase (TIMP)-1 expression, and has thus prompted the suggestion that CD40 signalling destabilizes plaques by promoting extracellular matrix (ECM) degradation and, as a result, fibrous cap thinning. ${ }^{6}$ Therefore, in this study we searched for similar associations in human samples. Plaque levels of CD40 correlated with plaque content of MMP-1, $-2,-9$, and -10 , while SCD40L levels correlated with only MMP-10 (Supplementary Table 6) 
Furthermore, both SCD40 and SCD40L levels correlated with TIMP-1 and TIMP-2. Thus, sCD40 (and CD40L) were indeed associated with plaque ECM turnover. Of note, correlations were also found between both SCD40 and SCD40L levels and content of intact elastin and collagen (type I-V) measured in plaque homogenate.

\section{Plaque sCD40 and SCD40L levels are not associated with pre-operative cerebrovascular symptoms}

As SCD40 and SCD40L plasma levels were higher in subjects who had suffered a stroke in the SUMMIT cohort, we next analysed whether SCD40 and/or SCD40L levels are similarly elevated in the plaques from individuals after a cerebrovascular event (median time between symptom and surgery was 14 days [IOR, 7 to 22]; the CPIP cohort). Levels of CD40 and CD40L measured in plaque strongly correlate with each other $(r=0.613, P<0.0001)$ but neither sCD40, nor sCD40L levels correlated with the time between symptoms exhibited by the patients and the time of endarterectomy $(r=0.04, P=0.688$ and $r=0.063, P=0.523$, respectively). Also, plaque levels of sCD40 and $\mathrm{SCD} 40 \mathrm{~L}$ were similar in plaques from asymptomatic and symptomatic patients ( $n=102$ and $n=96$, respectively).

\section{Discussion}

With this study, we set out to assess the value of sCD40 as a possible marker of CVD and investigate the biological associations that support a role for CD40 in atherogenesis. Our findings that sCD40 levels in plasma are associated with carotid plaque burden, while levels in carotid plaques are associated with a vulnerable plaque phenotype, strengthens the potential for SCD40 as marker for and mediator of CVD.

In contrast to the paucity of data in the literature for SCD40 as a marker for CVD, SCD40L has been previously widely linked to CVD via increased levels in patients with hypercholesterolemia $^{10}$ and acute coronary syndromes. ${ }^{11,24}$ Upregulated platelet surface expression of $\mathrm{CD} 40 \mathrm{~L}$ was also reported to correlate with worse clinical outcome after stroke ${ }^{25}$ and plasma sCD40L levels were higher in patients with carotid artery lesions that have intra-plaque lipid as detected by high-resolution magnetic resonance imaging. ${ }^{26}$ Furthermore, Balla et al. ${ }^{27}$ identified serum SCD40L levels as a risk factor for increased IMT in subjects with white-coat hypertension. Our current results are in line with these studies.

Much less is known about the possible role of sCD40 as a marker for CVD. Though SCD40 appears to be actively produced under physiological conditions (as suggested by comparatively large amounts of sCD40 found in urine of healthy individuals), ${ }_{1}^{28}$ elevated plasma levels of SCD40 has nonetheless been described in pathologies such as systemic lupus erythematosus $_{1}{ }^{29}$ slow coronary flow phenomenon ${ }^{30}$ and early Alzheimer's disease. ${ }^{31}$ In the SUMMIT cohort, we found higher plasma sCD40 levels in individuals that had suffered a prior ischemic stroke or $\mathrm{MI}$ or were hypertensive. Plasma SCD40 levels also correlated with carotid plaque burden and were associated with future CV events over a follow-up period of 3 years. We therefore propose $\mathrm{SCD} 40$ as a potential marker for the presence and severity of CVD.

Efficient targeting of inflammatory processes can successfully stabilize vulnerable plaques in mice $e^{8,9}$ but this approach is not yet utilized in human disease beyond clinical trials ${ }^{32}$ due to detrimental side effects. In atherosclerotic mouse models disrupted CD40 signalling-after blockade with antagonistic antibodies, small molecule inhibitors and in CD40- and CD40L-deficient mice-consistently results in plaques of reduced size and, importantly, a stabilized phenotype characterized by reduced lipid core content, macrophage and T-lymphocyte content accompanied by increased fibrous cap thickness, and relative collagen and SMC cell content. ${ }^{6-9}$ Previous studies exploring CD40 signalling in relation to severity of inflammatory diseases in humans have mainly focused on systemic levels of the signalling dyad, in particular levels of SCD40L and CD40L. This is the first study to also investigate associations between carotid intra-plaque CD40/CD40 content and plaque phenotype and disease progression. We found intra-plaque SCD40 (and to a certain extent also SCD40L) levels to be associated with a plaque profile rich in lipids, MMPs and pro-inflammatory cytokines and chemokines: destabilising elements corresponding to those affected by blocking of the CD40/CD40L axis in mice. ${ }^{6-9}$ This link between human disease and mouse model strengthens the merit of translating therapeutic targeting of CD40 from the academic setting one step closer to the clinical application.

Neither CD40 nor CD40L was associated with time between cerebrovascular symptoms and endarterectomy, suggesting they are not merely upregulated as part of the general healing response that occurs after plaque rupture. However, though sCD40 levels were associated with a less stable carotid plaque phenotype, plaque levels were nonetheless similar in plaques from symptomatic and asymptomatic patients. This inconsistency may, at least in part, be explained by the accompanying association with components of the ECM turnover machinery, leading to a net effect in CD40-rich plaques, where enough ECM synthesis of stable plaque elements balances out the more vulnerable elements, resulting in a plaque phenotype still 
stable enough not to rupture/erode and cause cerebrovascular symptoms. This in turn may indicate a local protective element in CD40-CD40L signalling, in addition to its well-described actions promoting the inflammatory process. We hypothesize that this may demonstrate the contrasting results of CD40 and CD40L interactions with (and in) different cell types of varying functions, such as macrophages, T cells, and SMCs in a model where CD40-signalling simultaneously for example promotes inflammation via macrophages and ECM synthesis via SMCs, even if the phenotypes of these cells have also been widely discussed. ${ }^{33}$ The therapeutic angle described by Seijkens et al. ${ }^{9}$ of targeting CD40 signalling blockade specifically to macrophages (via incorporation into reconstituted high-density lipoproteins nanoparticles) therefore also emerges as an especially relevant approach to achieve plaque stabilisation.

Also interesting from a therapeutic standpoint is the study by Fernandez et al., ${ }^{34}$ in which single cell RNA sequencing was compared in carotid plaques from patients who had or had not experienced cerebrovascular symptoms. Unexpectedly, the most upregulated pathways in T cells and macrophages from plaques from symptomatic patients were those of repair, while pro-inflammatory pathways were elevated in plaques from asymptomatic patients. Repair pathways are thus deduced to be induced by plaque rupture, and therefore represent a less striking presence in plaques whose integrity is more intact. Consequently, inflammatory activity weighs heavier in plaques from asymptomatic than from symptomatic individuals. This notion confirms the significance of our report that SCD40 is associated with vulnerable plaque components such as lipid content and pro-inflammatory cytokines, as well as with overall plaque burden, regardless of whether symptoms have manifested or not. Furthermore, the great success in ameliorating size and vulnerability of murine lesions (that are, per definition, pre-rupture) by blocking CD40 signalling affirms the value of targeting inflammatory responses in non-ruptured lesions.

As limitations, it must be noted that correlations do not indicate causality, and we cannot rule out that the reported associations may also result from an upregulation of SCD40 and sCD40L stimulated by an upstream signalling cascade or events. It is also important to bear in mind that in the plaque homogenates derived from the CPIP cohort it is not possible to distinguish between soluble and cell-bound CD40 and CD40L. The analysis thus shows the net effect of both isoforms, even if the effects of the individual isoforms may differ. Moreover, though it is impossible to be certain of the exact type of ischemic stroke, as subjects with atrial fibrillation were excluded in both cohorts, and-in the CPIP cohort-no signs of lacunar infarctions were found (assessed by brain computed tomogra-

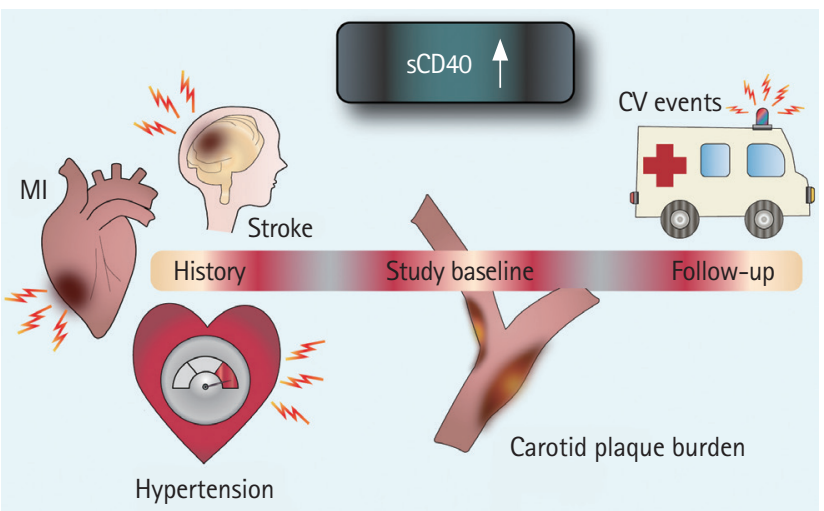

Figure 3. Graphic representation of plasma soluble CD40 (sCD40) in cardiovascular disease. Higher plasma SCD40 levels are associated with prevalent cardiovascular disease, severity of carotid atherosclerosis and predict future cardiovascular events (CV events). MI, myocardial infarction.

phy), the probability of the underlying mechanisms to be atrial fibrillation and small vessel disease rather than atherosclerosis can be considered low. Unfortunately, the presence of cardiac valvular disease is impossible to exclude as not all patients underwent echocardiography. Finally, as we in both cohorts measured SCD40L in plasma to evaluate its potential as a non-cell associated marker, platelet expression of CD40L-previously reported e.g., by Cha et al. ${ }^{35}$ to be higher in subjects with atherosclerotic ischemic stroke than with asymptomatic carotid stenosis-has not been taken into account.

\section{Conclusions}

In the present study we report for the first time elevated plasma sCD40 levels in individuals with a prior ischemic stroke or $\mathrm{MI}$, as well as an association with arterial stiffness, carotid plaque burden, and IMT, and, finally, an association with increased risk of future CV events (Figure 3). Plasma levels of sC$\mathrm{D} 40 \mathrm{~L}$ are confirmed to not only be elevated in subjects after stroke, but also to correlate with future plaque progression in the form of IMT increase. We also present a novel association between intra-plaque levels of CD40 and CD40L and a vulnerable plaque phenotype.

\section{Supplementary materials}

Supplementary materials related to this article can be found online at https://doi.org/10.5853/jos.2021.00178.

\section{Disclosure}

The authors have no financial conflicts of interest. 


\section{Acknowledgments}

This work was supported by funding from the Innovative Medicines Initiative (the SUMMIT consortium), IMI-2008/115006, Swedish Research Council, Swedish Heart and Lung Foundation, Swedish Society for Medical Research, Swedish Society of Medicine, Emil and Wera Cornell foundation, Hjelt foundation, ALF Grants Region Skane, Bundy Academy, Diabetes foundation, Diabetes Research \& Wellness Foundation, SUS foundations and funds, Stroke foundation, the Royal Physiographic Society in Lund, The Swedish Heart and Lung Association, The Swedish Stroke Association, Albert Påhlsson's foundation and Swedish Foundation for Strategic Research (Dnr IRC15-0067). Angela C. Shore is supported by the UK National Institute of Health Research (NIHR) Exeter Clinical Research Facility. The views in this manuscript are those of the authors and not necessarily those of the NIHR or the UK Department of Health.

The authors wish to thank Mihaela Nitulescu, Ana Persson, Lena Sundius, and Fong To for expert technical assistance.

\section{References}

1. World Health Organization. Cardiovascular Disease Fact Sheet. Geneva, CH: World Health Organization, 2017.

2. Sakakura K, Nakano M, Otsuka F, Ladich E, Kolodgie FD, Virmani R. Pathophysiology of atherosclerosis plaque progression. Heart Lung Circ 2013;22:399-411.

3. Hansson GK, Libby P, Tabas I. Inflammation and plaque vulnerability. J Intern Med 2015;278:483-493.

4. Noelle RJ, Ledbetter JA, Aruffo A. CD40 and its ligand, an essential ligand-receptor pair for thymus-dependent B-cell activation. Immunol Today 1992;13:431-433.

5. Jobling K, Ng WF. CD40 as a therapeutic target in Sjögren's syndrome. Expert Rev Clin Immunol 2018;14:535-537.

6. Lutgens E, Lievens D, Beckers L, Wijnands E, Soehnlein O, Zernecke $A$, et al. Deficient CD40-TRAF6 signaling in leukocytes prevents atherosclerosis by skewing the immune response toward an antiinflammatory profile. J Exp Med 2010; 207:391-404.

7. Lutgens E, Gorelik L, Daemen MJ, de Muinck ED, Grewal IS, Koteliansky VE, et al. Requirement for CD154 in the progression of atherosclerosis. Nat Med 1999;5:1313-1316.

8. Lutgens $E_{1}$ Cleutjens KB, Heeneman S, Koteliansky VE, Burkly LC, Daemen MJ. Both early and delayed anti-CD40L antibody treatment induces a stable plaque phenotype. Proc Natl Acad SciUSA 2000;97:7464-7469.

9. Seijkens TTP, van Tiel CM, Kusters PJH, Atzler D, Soehnlein $O_{\text {, }}$ Zarzycka B, et al. Targeting CD40-induced TRAF6 signaling in macrophages reduces atherosclerosis. J Am Coll Cardiol 2018;71:527-542.

10. Garlichs CD, John S, Schmeisser A, Eskafi S, Stumpf C, Karl $M$, et al. Upregulation of CD40 and CD40 ligand (CD154) in patients with moderate hypercholesterolemia. Circulation 2001;104:2395-2400.

11. Heeschen C, Dimmeler S, Hamm CW, van den Brand MJ, Boersma $E$, Zeiher AM, et al. Soluble CD40 ligand in acute coronary syndromes. N Engl J Med 2003;348:1104-1111.

12. Schönbeck U, Varo N, Libby P, Buring J, Ridker PM. Soluble $\mathrm{CD} 40 \mathrm{~L}$ and cardiovascular risk in women. Circulation 2001;104:2266-2268.

13. Huang YQ, Jie $L I$, Chen JY, Tang ST, Huang $C$, Feng YQ. The relationship between soluble CD40 ligand level and atherosclerosis in white-coat hypertension. J Hum Hypertens 2017; 32:40-45.

14. Lobbes MB, Lutgens $E_{1}$ Heeneman $S$, Cleutjens $K B$, Kooi ME, van Engelshoven $\mathrm{JM}$, et al. Is there more than C-reactive protein and fibrinogen? The prognostic value of soluble CD40 ligand, interleukin-6 and oxidized low-density lipoprotein with respect to coronary and cerebral vascular disease. Atherosclerosis 2006;187:18-25.

15. de Lemos JA, Zirlik A, Schönbeck U, Varo N, Murphy SA, Khera $A$, et al. Associations between soluble CD40 ligand, atherosclerosis risk factors, and subclinical atherosclerosis: results from the Dallas Heart Study. Arterioscler Thromb Vasc Biol 2005;25:2192-2196.

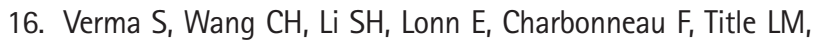
et al. The relationship between soluble CD40 ligand levels and Framingham coronary heart disease risk score in healthy volunteers. Atherosclerosis 2005;182:361-365.

17. Gergei I, Kälsch T, Scharnagl H, Kleber ME, Zirlik $A$, März W, et al. Association of soluble CD40L with short-term and long-term cardiovascular and all-cause mortality. The Ludwigshafen Risk and Cardiovascular Health (LURIC) study. Atherosclerosis 2019;291:127-131.

18. Shore AC, Colhoun HM, Natali A, Palombo C, Östling G, Aizawa $\mathrm{K}$, et al. Measures of atherosclerotic burden are associated with clinically manifest cardiovascular disease in type 2 diabetes: a European cross-sectional study. J Intern Med 2015;278:291-302.

19. Asciutto $G$, Edsfeldt A, Dias NV, Nilsson J, Prehn C, Adamski $J$, et al. Treatment with beta-blockers is associated with lower levels of Lp-PLA2 and suPAR in carotid plaques. Cardiovasc Pathol 2013;22:438-443.

20. Kim HL, Kim SH. Pulse wave velocity in atherosclerosis. Front Cardiovasc Med 2019;6:41.

21. Companjen AR, van der Wel LI, Boon L, Prens EP, Laman JD. 
CD40 ligation-induced cytokine production in human skin explants is partly mediated via IL-1. Int Immunol 2002;14: 669-676.

22. Basok A, Shnaider A, Man L, Chaimovitz C, Douvdevani A. CD40 is expressed on human peritoneal mesothelial cells and upregulates the production of interleukin-15 and RANTES. $J$ Am Soc Nephrol 2001;12:695-702.

23. Buhtoiarov IN, Lum $H$, Berke G, Paulnock DM, Sondel PM, Rakhmilevich AL. CD40 ligation activates murine macrophages via an IFN-gamma-dependent mechanism resulting in tumor cell destruction in vitro. J Immunol 2005;174:60136022.

24. Fouad HH, Al-Dera $H$, Bakhoum SW, Rashed LA, Sayed RH, Rateb MA, et al. Levels of SCD40 ligand in chronic and acute coronary syndromes and its relation to angiographic extent of coronary arterial narrowing. Angiology 2010;61:567-573.

25. Lukasik M, Dworacki G, Kufel-Grabowska J, Watala C, Kozubski W. Upregulation of CD40 ligand and enhanced monocyte-platelet aggregate formation are associated with worse clinical outcome after ischaemic stroke. Thromb Haemost 2012; 107:346-355.

26. Blake GJ, Ostfeld RJ, Yucel EK, Varo N, Schönbeck U, Blake MA, et al. Soluble CD40 ligand levels indicate lipid accumulation in carotid atheroma: an in vivo study with high-resoIution MRI. Arterioscler Thromb Vasc Biol 2003;23:e11-e14.

27. Balla J, Magyar MT, Bereczki D, Valikovics A, Nagy E, Barna $E_{1}$ et al. Serum levels of platelet released CD40 ligand are increased in early onset occlusive carotid artery disease. Dis Markers 2006;22:133-140.

28. Contin C, Pitard V, Delmas Y, Pelletier N, Defrance T, Moreau $\mathrm{JF}$, et al. Potential role of soluble CD40 in the humoral im- mune response impairment of uraemic patients. Immunology 2003;110:131-140.

29. Bae SC, Lee YH. Association between CD40 polymorphisms and systemic lupus erythematosus and correlation between soluble CD40 and CD40 ligand levels in the disease: a meta-analysis. Lupus 2019;28:1452-1459.

30. Durakoğlugil ME, Kocaman $S A$, Çetin $M$, Kirbas $A$, Canga $A$, Erdoğan $T$, et al. Increased circulating soluble CD40 levels in patients with slow coronary flow phenomenon: an observational study. Anadolu Kardiyol Derg 2013;13:39-44.

31. Buchhave $P$, Janciauskiene $S$, Zetterberg $H$, Blennow $K$, Minthon L, Hansson 0. Elevated plasma levels of soluble CD40 in incipient Alzheimer's disease. Neurosci Lett 2009; 450:56-59.

32. Ridker PM, Everett BM, Thuren $T$, MacFadyen JG, Chang WH, Ballantyne $C$, et al. Antiinflammatory therapy with canakinumab for atherosclerotic disease. N Engl J Med 2017;377: 1119-1131.

33. Wirka RC, Wagh D, Paik DT, Pjanic M, Nguyen T, Miller $C L$, et al. Atheroprotective roles of smooth muscle cell phenotypic modulation and the TCF21 disease gene as revealed by single-cell analysis. Nat Med 2019;25:1280-1289.

34. Fernandez DM, Rahman AH, Fernandez NF, Chudnovskiy A, Amir ED, Amadori $L$, et al. Single-cell immune landscape of human atherosclerotic plaques. Nat Med 2019;25:15761588.

35. Cha JK, Jeong MH, Jang JY, Bae HR, Lim YJ, Kim JS, et al. Serial measurement of surface expressions of CD63, P-selectin and CD40 ligand on platelets in atherosclerotic ischemic stroke. A possible role of CD40 ligand on platelets in atherosclerotic ischemic stroke. Cerebrovasc Dis 2003;16:376-382. 


\section{Supplementary methods}

\section{Study cohorts}

The studies were approved by the local ethical review boards and were carried out in accordance with the principles of the Declaration of Helsinki. All study subjects provided written informed consent. There was no overlap between subjects of the two study cohorts.

\section{The SUMMIT}

The SUrrogate markers for Micro- and Macro-vascular hard endpoints for Innovative diabetes Tools (SUMMIT) study ${ }^{1}$ population included 1,438 subjects recruited between December 2010 and April 2013 from existing population cohorts and hospital registries at the university hospitals in Malmö (Sweden), Pisa (Italy), Dundee and Exeter (UK)-62 subjects were excluded (from the original cohort, $n=1,500$ ), due to insufficient quality of CD40/CD40L Proximity Extension Assay (PEA) results (Figure 1). Clinical characteristics of the included patients are summarized in Supplementary Table 1. Diabetes was defined by contemporary or historical evidence of hyperglycaemia (according to World Health Organization 1998 criteria; fasting plasma glucose $>7.0 \mathrm{mmol} / \mathrm{L}$ or 2-hour plasma glucose $>11.1 \mathrm{mmol} / \mathrm{L}$, or both) or by current medication with insulin, sulphonylureas, metformin or other anti-diabetic drugs. To define type 2 diabetes, patients should have been diagnosed after the age of 30 . Subjects diagnosed $<35$ years of age or treated with insulin within 12 months of diagnosis were excluded. Classification of a clinical history of cardiovascular disease included a previous diagnosis in the clinical record of non-fatal acute myocardial infarction (AMI), hospitalized unstable angina, resuscitated cardiac arrest, any coronary revascularization procedure, non-fatal stroke, transient ischemic attack confirmed by a specialist, and lower extremities arterial disease (LEAD) defined as Ankle Brachial Pressure Index (ABPI) $<0.9$ with intermittent claudication or prior corrective surgery, angioplasty or above ankle amputation. Exclusion criteria included renal replacement therapy, malignancy requiring active treatment, end-stage renal disease, any chronic inflammatory disease on therapy, previous bilateral carotid artery invasive interventions, or age $<40$ years. Additionally, subjects with atrial fibrillation were excluded. Demographics, clinical characteristics including medication, physical and laboratory examinations were obtained according to a pre-defined study protocol at all four participating centers.

\section{The CPIP}

Included in the Carotid Plaque Imaging Project (CPIP) study ${ }^{2}$ were 198 carotid plaques collected from patients during carotid endarterectomies at the Vascular Department of Skane University Hospital (Malmö, Sweden) between 2005 and 2010. Clinical characteristics of the included patients are summarized in Supplementary Table 1. Blood samples were taken the day before endarterectomy.

Surgery indications have been described previously. ${ }^{2}$ Briefly, symptomatic patients exhibited carotid artery stenosis (>70\%) associated with ipsilateral symptoms, and asymptomatic patients exhibited carotid stenosis $(>80 \%)$, but no ipsilateral symptoms 6 months prior to endarterectomy. Symptoms (i.e., amaurosis fugax, transient ischemia attack, or stroke) were evaluated by a neurologist and the stenosis degree was evaluated by ultrasound based on flow velocities as previously described. ${ }^{3}$ Patients with atrial fibrillation were excluded.

Immediately after surgery, removed plaques were snap-frozen in liquid nitrogen. Histological analyses were performed on portions (1 $\mathrm{mm}$ thick) cut from the most stenotic plaque region and embedded in optimal cutting medium (Sakura Finetek Europe $B V$, Tokyo, Japan) and then sectioned $(8 \mu \mathrm{m})$. Plaque homogenates were prepared from the rest of the snap-frozen plaques and used for all other plaque component quantifications as previously described. ${ }^{4}$

\section{Plasma analysis}

CD40 and CD40L were analysed in plaque homogenates from the CPIP cohort and in plasma from SUMMIT cohort using the PEA technique using the Proseek Multiplex CVD96x96 reagents kit (Olink Bioscience, Uppsala, Sweden) as described previously. ${ }^{5}$

\section{The SUMMIT cohort: ultrasound imaging and evaluation of arterial stiffness}

Atherosclerosis status was assessed via ultrasound imaging of the carotid arteries examining intima-media thickness (IMT) in the common carotid artery (CCA) and carotid bulb. Plaques were defined as focal thickenings $(\geq 0.8 \mathrm{~mm})$ of the artery wall. The length and height of each individual plaque were measured to calculate plaque area. The inter-observer variability of plaque area measurements was $8.9 \% \pm 4.6 \%$. The total plaque area represents the sum of the area all plaques identified in the left and right carotid arteries. Pulse wave velocity as an assessment for arterial stiffness was calculated using a Sphygmocor device (Atcor Medical, Sydney, Australia). Methods were performed as described previously. ${ }^{6}$ Change in mean and maximal IMT in the CCA and bulb (in mm) at the follow-up point is expressed as the measurements taken at inclusion subtracted by measurements taken at follow-up. 


\section{The CPIP cohort: histology}

Histological/immunohistochemical detection of Oil Red 0, alpha smooth muscle actin, $\mathrm{CD} 68{ }_{1}^{7.8}$ and glycophorin $\mathrm{A}^{9}$ was performed as previously described on frozen tissue sections. Collagen was visualized through a standard Russel-Movat pentachrome stain. For the Von Kossa calcium stain sections were fixed in 100\% ethanol and then incubated in 1\% silver nitrate, $5 \%$ sodium thiosulfate, and $0.1 \%$ fast nuclear red. Between incubations sections were rinsed in distilled $\mathrm{H}_{2} \mathrm{O}$. Stained slides were scanned and digitalized using an Aperio ScanScope digital slide scanner (Aperio Technologies Inc., Vista, CA, USA). Quantifications were performed on blinded samples, using BioPix iQ version 2.3.1 imaging software (Biopix AB, Gothenburg, Sweden). Vulnerability index was calculated as

\footnotetext{
$(\mathrm{CD} 68+)+($ Glycophorin $\mathrm{A}+)+($ Oil Red $0+)$ area\%

$($ Smooth muscle a-actin +$)+($ Collagen +$)+($ von Kossa +$)$ area\% .
}

Paraffin-embedded sections from 22 subjects were used for immunofluorescence detection of CD40 and CD40L. Sections was deparaffinised and re-hydrated in a graded series of ethanol. Heat-induced epitope retrieval ( $\mathrm{pH} \mathrm{6)}$ was performed prior to incubation with primary antibodies for 1 hour (CD40: ab13545, CD40L: ab2391; Abcam, Cambridge, UK). As a negative control an isotype control (ab27478, Abcam) of matching antibody concentration and host species was used. Sections were incubated with an Alexa 555-conjugated secondary antibody (ab150074, Abcam) for 1 hour and then mounted with a DAPI-containing mounting media (Vectashield Antifade mounting medium with DAPI, Vector Laboratories Inc., Burlingtame, CA, USA). Images were taken using a Nikon Eclipse E800 microscope (BergmanLabora, Danderyd, Sweden) with Olympus cellSens Standard 1.18 software.

\section{The CPIP cohort: plaque component analyses on} plaque homogenates

Multiplex analysis of cytokines (interleukin [IL]-6, IL-23p70, C-X-C motif chemokine ligand 1 [CXCL-1], chemokine (C-C motif) ligand $[\mathrm{CCL}]-2,-4$, and -5 , platelet-derived growth factor $\mathrm{AA} / \mathrm{BA} / \mathrm{BB}$, and tumor necrosis factor- $\mathrm{a}$ ) was performed using Luminex as previously described. ${ }^{9}$ Matrix metalloproteinases (MMPs) $-1,-2,-9,-10$ and tissue inhibitor of metalloproteinases (TIMPS) $-1,-2$, and -3 were measured using the Mesoscale human MMP ultra-sensitive kit (Mesoscale, Gaithersburg, MD, USA) and MILLIPLEX MAP Human TIMP Magnetic Bead Panel (Milliplex, Millipore Corporation, Billerica, MA, USA), respectively, as previously described. ${ }^{9}$ Oxidized low density lipoprotein was analysed by enzyme-linked immunosorbent assay (ELISA) as previously described. ${ }^{10}$ Collagen and elastin were quantified using the Sircol soluble collagen assay (Biocolor, Carrickfergus, UK) and the Fastin elastin assay (Biocolor), respectively, as described previously. ${ }^{4}$ Results were normalized to plaque wet weight and all analyses were performed according to the manufacturer's instructions.

\section{Statistics}

Shapiro-Wilk and D'Agostino-Pearson omnibus K2 tests were used to assess Gaussian distribution. Variables found to be normally distributed are shown as mean with standard deviation while non-normally distributed variables are shown as median with interquartile range. For analysis of plasma, plaque histology sections and homogenates, the Mann-Whitney $U$ test was used to compare groups and Spearman's rank correlation was used for continuous variables. For comparisons between categorical variables, the chi-square test was used. Spearman's rank correlation was chosen (over Pearson's correlation test) due to the non-Gaussian distribution of the CD40 and CD40L variables and to account for monotonic as well as linear relationships between $\mathrm{CD} 40 \mathrm{~L}$ and the various parameters tested.

Subjects included in the SUMMIT cohort were followed up after 36 months at all four sites and information of cardiovascular events were registered. Cardiovascular events were defined as AMI (non-fatal or fatal), hospitalized unstable angina, cardiac arrest (resuscitated or fatal), any coronary revascularization procedure, stroke (non-fatal or fatal), and transient ischemic attack confirmed by a specialist, LEAD defined as $A B P I<0.9$ with intermittent claudication or prior corrective surgery, angioplasty, or above ankle amputation. A logistic regression model was used to test for associations with cardiovascular events and mortality during a follow-up. The model was adjusted for age, diabetes and prevalent stroke, AMI, and hypertension as covariates. The model was implemented in $\mathrm{R}$ using the rms package. ${ }^{11}$ Area under the receiver operating characteristics (ROC) curve and confidence intervals were estimated by the pROC package ${ }^{12}$ with 2,000 stratified bootstrap replicates. To further examine performances of our proposed model in decision making, sensitivities and specificities with confidence intervals were estimated by the pROC package ${ }^{12}$ with 2,000 stratified bootstrap replicates using varying cut-off values of the predicted values from the logistic regression. Considering that the costs of false positives and false negatives may vary in clinical applications, we examined sensitivities and specificities from $10 \%$ to $90 \%$ quantile of the predicted values. A high specificity level would be preferred if less false positives were expected.

A $P$-value of $<0.05$ was considered statistically significant. Statistical analysis was performed using SPSS version 26.0 
(IBM Co., Armonk, NY, USA) and GraphPad Prism version 8.0.0 for Windows (GraphPad Software, San Diego, CA, USA, www. graphpad.com). Adjustments for multiple comparisons were done when appropriate using the Holm-Šíák test.

\section{Supplementary References}

1. Shore AC, Colhoun HM, Natali A, Palombo C, Östling G, Aizawa $K$, et al. Measures of atherosclerotic burden are associated with clinically manifest cardiovascular disease in type 2 diabetes: a European cross-sectional study. J Intern Med 2015;278: 291-302.

2. Asciutto G, Edsfeldt A, Dias NV, Nilsson J, Prehn C, Adamski $J$, et al. Treatment with beta-blockers is associated with lower levels of Lp-PLA2 and suPAR in carotid plaques. Cardiovasc Pathol 2013;22:438-443.

3. Hansen $F$, Bergqvist $D$, Lindblad B, Lindh $M$, Mätzsch T, Länne T. Accuracy of duplex sonography before carotid endarterectomy: a comparison with angiography. Eur J Vasc Endovasc Surg 1996;12:331-336.

4. Gonçalves I, Moses J, Dias N, Pedro LM, Fernandes e Fernandes J, Nilsson J, et al. Changes related to age and cerebrovascular symptoms in the extracellular matrix of human carotid plaques. Stroke 2003;34:616-622.

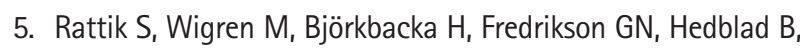
Siegbahn $A$, et al. High plasma levels of heparin-binding epidermal growth factor are associated with a more stable plaque phenotype and reduced incidence of coronary events.
Arterioscler Thromb Vasc Biol 2015;35:222-228.

6. Gonçalves I, Bengtsson E, Colhoun HM, Shore AC, Palombo C, Natali A, et al. Elevated plasma levels of MMP-12 are associated with atherosclerotic burden and symptomatic cardiovascular disease in subjects with type 2 diabetes. Arterioscler Thromb Vasc Biol 2015;35:1723-1731.

7. Gonçalves I, Edsfeldt A, Ko NY, Grufman H, Berg K, Björkbacka $\mathrm{H}$, et al. Evidence supporting a key role of Lp-PLA2-generated lysophosphatidylcholine in human atherosclerotic plaque inflammation. Arterioscler Thromb Vasc Biol 2012;32: 1505-1512.

8. Edsfeldt A, Nitulescu M, Grufman H, Grönberg C, Persson A, Nilsson $M$, et al. Soluble urokinase plasminogen activator receptor is associated with inflammation in the vulnerable human atherosclerotic plaque. Stroke 2012;43:3305-3312.

9. Shami A, Tengryd C, Asciutto G, Bengtsson E, Nilsson J, Hultgårdh-Nilsson $A$, et al. Expression of fibromodulin in carotid atherosclerotic plaques is associated with diabetes and cerebrovascular events. Atherosclerosis 2015;241:701-708.

10. Dunér $P$, Gonçalves I, Grufman $H$, Edsfeldt $A$, To $F$, Nitulescu $M$, et al. Increased aldehyde-modification of collagen type IV in symptomatic plaques: a possible cause of endothelial dysfunction. Atherosclerosis 2015;240:26-32.

11. Harrell FEJ. Package 'rms'. https://cran.r-project.org/web/ packages/rms/rms.pdf. 2021. Accessed July 3, 2021.

12. Robin X, Turck N, Hainard A, Tiberti N, Lisacek F, Sanchez JC, et al. pROC: an open-source package for $\mathrm{R}$ and $\mathrm{S}+$ to analyze and compare ROC curves. BMC Bioinformatics 2011;12:77. 
Supplementary Table 1. Patient demographics in the CPIP and SUMMIT study cohorts

\begin{tabular}{|c|c|c|c|c|}
\hline Variable & CPIP $(n=198)$ & SUMMIT $(n=1,438)$ & $P$ & $P$, adjusted* \\
\hline \multicolumn{5}{|l|}{ At baseline } \\
\hline Sex (\% female) & 32 & 35 & 0.43 & 0.81 \\
\hline Age $(\mathrm{yr})$ & $70(64.2-75.8)$ & $68(62-74)$ & 0.0034 & 0.020 \\
\hline Body mass index $\left(\mathrm{kg} / \mathrm{m}^{2}\right)$ & $27.3 \pm 4.1$ & $28.6(25.6-32.3)$ & $1.9 \times 10^{-12}$ & $2.1 \times 10^{-11}$ \\
\hline Smoking, currently or in the past $(\%)$ & 80 & 62 & $1.9 \times 10^{-5}$ & 0.00015 \\
\hline Diabetes (\%) & 33 & 66 & $<1 \times 10^{-16}$ & $<1 \times 10^{-14}$ \\
\hline Hypertension (\%) & 75 & 67 & 0.028 & 0.11 \\
\hline Use of statins (\%) & 88 & 66 & $5.7 \times 10^{-10}$ & $5.1 \times 10^{-9}$ \\
\hline Under treatment $(\%)^{+}$ & 97 & 80 & $<1 \times 10^{-16}$ & $<1 \times 10^{-14}$ \\
\hline \multicolumn{5}{|l|}{ Fasting lipoproteins (mmol/L) } \\
\hline Total cholesterol & $4.1(3.5-5.1)$ & $4.2(3.6-5.0)$ & 0.69 & 0.81 \\
\hline Triglycerides & $1.4(1-1.8)$ & $1.3(1.0-1.9)$ & 0.50 & 0.81 \\
\hline LDL-C & $2.4(1.8-3.1)$ & $2.3(1.8-3.0)$ & 0.018 & 0.087 \\
\hline HDL-C & $1.0(0.9-1.3)$ & $1.3(1.0-1.5)$ & $1.8 \times 10^{-11}$ & $1.8 \times 10^{-10}$ \\
\hline $\mathrm{HbA1c}(\mathrm{mmol} / \mathrm{mol})$ & $43.9(38-55)$ & $48(40-58.3)$ & 0.0025 & 0.017 \\
\hline Hemoglobin $(g / L)$ & $140.7 \pm 13.3$ & - & - & - \\
\hline White blood cell counts $\left(\times 10^{9} / \mathrm{L}\right)$ & $7.8(6.4-9.2)$ & - & - & - \\
\hline Platelet count $\left(\times 10^{9} / \mathrm{L}\right)$ & 249 (207-298) & - & - & - \\
\hline $\mathrm{CRP}(\mathrm{mg} / \mathrm{L})$ & $3.9(2.0-6.4)$ & $1.4(0.68-3.0)$ & $<1 \times 10^{-16}$ & $<1 \times 10^{-14}$ \\
\hline CD40 (A.U..$\left.^{\ddagger}\right)$ & $3.2 \times 10^{7}\left(2.4 \times 10^{7}-4.04 \times 10^{7}\right)$ & $9.1(8.7-9.9)$ & $<1 \times 10^{-16}$ & $<1 \times 10^{-14}$ \\
\hline CD40L (A.U. $)$ & $1.8 \times 10^{5}\left(1.3 \times 10^{5}-2.6 \times 10^{5}\right)$ & $6.8(5.4-8.2)$ & $<1 \times 10^{-16}$ & $<1 \times 10^{-14}$ \\
\hline
\end{tabular}

Values are presented as median (interquartile range) or mean \pm standard deviation. Categorical variables were compared using the chi-square test and continuous variables by the Mann-Whitney U test or unpaired t-test (for non-normally and normally distributed samples, respectively).

CPIP, Carotid Plaque Imaging Project; SUMMIT, The SUrrogate markers for Micro- and Macro-vascular hard endpoints for Innovative diabetes Tools; LDL-C, low density lipoprotein cholesterol; HDL-C, high density lipoprotein cholesterol; HbA1c, hemoglobin A1c; CRP, C-reactive protein; A.U., arbitrary unit; CD40L, CD40 ligand.

${ }^{*} P$-value adjusted for multiple comparisons (according to the Holm-Šídák test); ${ }^{+}$Previous vascular intervention (percutaneous coronary intervention, coronary artery bypass grafting or drugs (statins, anticoagulant, beta-blocker, anti-hypertensive, renin-angiotensin-aldosterone system inhibitors); ${ }^{\ddagger}$ In CPIP relative to plaque wet weight.

Supplementary Table 2. Spearman correlations between sCD40 and sCD40L in plasma (arbitrary unit) and the subjects' general clinical characteristics (SUMMIT cohort)

\begin{tabular}{|c|c|c|c|c|c|c|}
\hline \multirow{2}{*}{ Variable } & \multicolumn{3}{|c|}{$\operatorname{CD} 40(n=1,438)$} & \multicolumn{3}{|c|}{ CD40L $(n=1,438)$} \\
\hline & $r$ & $P$ & $P$, adjusted & r & $P$ & $P$, adjusted \\
\hline Age (yr) & 0.399 & $<1 \times 10^{-16}$ & $<1 \times 10^{-16}$ & 0.234 & $<1 \times 10^{-16}$ & $<1 \times 10^{-16}$ \\
\hline Body mass index $\left(\mathrm{kg} / \mathrm{m}^{2}\right)$ & 0.002 & 0.93 & 0.94 & -0.124 & 0.000003 & 0.000018 \\
\hline \multicolumn{7}{|l|}{ Blood (mmol/L) } \\
\hline $\mathrm{HbA} 1 \mathrm{c}(\mathrm{mmol} / \mathrm{mol})$ & 0.015 & 0.58 & 0.91 & -0.006 & 0.81 & 0.85 \\
\hline f-Glucose & 0.133 & 0.00025 & 0.0015 & 0.131 & 0.00033 & 0.0020 \\
\hline Total cholesterol & -0.061 & 0.022 & 0.11 & 0.109 & 0.000039 & 0.00023 \\
\hline LDL & -0.028 & 0.31 & 0.77 & 0.189 & $2.2 \times 10^{-12}$ & $1.76 \times 10^{-11}$ \\
\hline $\mathrm{HDL}$ & -0.001 & 0.97 & 0.97 & 0.076 & 0.004 & 0.016 \\
\hline Triglycerides & 0.015 & 0.56 & 0.91 & -0.070 & 0.008 & 0.024 \\
\hline
\end{tabular}

SCD40, soluble CD40; SCD40L, soluble CD40 ligand; SUMMIT, The SUrrogate markers for Micro- and Macro-vascular hard endpoints for Innovative diabetes Tools; HbA1c, hemoglobin A1c; LDL, low density lipoprotein; HDL, high density lipoprotein. 
Supplementary Table 3. Median with IQR values of SCD40 and SCD40L in plasma (A.U., SUMMIT cohort) when comparing between two groups (MannWhitney U test)

\begin{tabular}{|c|c|c|c|c|}
\hline & \multicolumn{2}{|l|}{ CD40 $(n=1,438)$} & \multicolumn{2}{|l|}{$\operatorname{CD} 40 \mathrm{~L}(\mathrm{n}=1,438)$} \\
\hline & Content (A.U.) & $P$, adjusted ${ }^{*}$ & Content (A.U.) & $P$, adjusted \\
\hline Female sex (vs. male sex) & 9.16 (8.71-9.90) vs. 9.14 (8.70-9.89) & 0.94 & 6.37 (5.04-8.06) vs. 6.99 (5.62-8.24) & 0.0016 \\
\hline Diabetes type 2 (yes vs. no) & 9.18 (8.74-9.91) vs. 9.08 (8.61-9.84) & 0.016 & 6.94 (5.48-8.27) vs. 6.52 (5.17-8.02) & 0.020 \\
\hline Stroke (yes vs. no) & 9.75 (9.08-10.37 ) vs. 9.12 (8.70-9.80) & 0.000030 & 7.88 (5.62-8.65) vs. 6.75 (5.37-8.16) & 0.020 \\
\hline Hypertension (yes vs. no) & 9.24 (8.73-9.95) vs. 8.98 (8.37-9.76) & 0.00021 & 6.86 (5.39-8.21) vs. 6.63 (5.33-8.18) & 0.51 \\
\hline AMI (yes vs. no) & 9.27 (8.70-9.98) vs. 9.10 (8.70-9.82) & 0.016 & 7.13 (5.47-8.24) vs. 6.71 (5.34-8.19) & 0.28 \\
\hline
\end{tabular}

IQR, interquartile range; SCD40, soluble CD40; sCD40L, soluble CD40 ligand; A.U., arbitrary unit; SUMMIT, The SUrrogate markers for Micro- and Macro-vascular hard endpoints for Innovative diabetes Tools; AMI, acute myocardial infarction.

${ }^{*} P$-value adjusted for multiple comparisons (according to the Holm-Šídák test).

Supplementary Table 4. Spearman correlations between the circulating levels of sCD40 and SCD40L (A.U., SUMMIT cohort) and change in IMT in the CCA and the carotid bulb region assessed by vascular ultrasound (at the 3-year follow-up point compared to study inclusion)

\begin{tabular}{|c|c|c|c|c|c|c|}
\hline \multirow[t]{2}{*}{ IMT change (mm) } & \multicolumn{3}{|c|}{$\begin{array}{c}\operatorname{CD} 40 \\
\text { ( } n=1,438 \text { at inclusion; } n=798 \text { at follow-up) }\end{array}$} & \multicolumn{3}{|c|}{$\begin{array}{c}\text { CD40L } \\
(\mathrm{n}=1,438 \text { at inclusion; } \mathrm{n}=798 \text { at follow-up) }\end{array}$} \\
\hline & $r$ & $P$ & $P$, adjusted ${ }^{*}$ & $r$ & $P$ & $P$, adjusted \\
\hline \multicolumn{7}{|l|}{ Right } \\
\hline Mean IMT in CCA & -0.046 & 0.18 & 0.63 & 0.014 & 0.69 & 0.90 \\
\hline Max IMT in CCA & 0.015 & 0.67 & 0.82 & 0.090 & 0.009 & 0.027 \\
\hline Mean IMT in bulb & 0.027 & 0.44 & 0.82 & 0.149 & 0.000022 & 0.00011 \\
\hline Max IMT in bulb & 0.075 & 0.034 & 0.21 & 0.243 & $3.65 \times 10^{-12}$ & $2.55 \times 10^{-11}$ \\
\hline \multicolumn{7}{|l|}{ Left } \\
\hline Mean IMT in CCA & -0.039 & 0.25 & 0.68 & 0.014 & 0.69 & 0.90 \\
\hline Max IMT in CCA & 0.051 & 0.14 & 0.59 & 0.115 & 0.001 & 0.0040 \\
\hline Mean IMT in bulb & 0.025 & 0.47 & 0.82 & 0.233 & $1.66 \times 10^{-10}$ & $9.96 \times 10^{-10}$ \\
\hline Max IMT in bulb & 0.092 & 0.009 & 0.070 & 0.261 & $7.86 \times 10^{-14}$ & $6.29 \times 10^{-13}$ \\
\hline
\end{tabular}

sCD40, soluble CD40; sCD40L, soluble CD40 ligand; A.U., arbitrary unit; SUMMIT, The SUrrogate markers for Micro- and Macro-vascular hard endpoints for Innovative diabetes Tools; IMT, intima-media thickness; CCA, common carotid artery.

${ }^{*} P$-value adjusted for multiple comparisons. 
Supplementary Table 5. Sensitivities, specificities and their 95\% Cl at various cut-offs of predicted values from LR in predicting atherosclerotic vascular events

\begin{tabular}{|c|c|c|c|c|c|c|c|}
\hline \multirow{2}{*}{ Quantile } & \multirow{2}{*}{$\begin{array}{l}\text { Cut-off of } \\
\text { predicted value }\end{array}$} & \multicolumn{3}{|c|}{ Sensitivity } & \multicolumn{3}{|c|}{ Specificity } \\
\hline & & Median & 95\% Cl lower & $95 \% \mathrm{Cl}$ upper & Median & 95\% Cl lower & 95\% $\mathrm{Cl}$ upper \\
\hline $10 \%$ & 0.060 & 0.980 & 0.953 & 1.000 & 0.110 & 0.093 & 0.128 \\
\hline $20 \%$ & 0.070 & 0.899 & 0.852 & 0.946 & 0.212 & 0.191 & 0.235 \\
\hline $30 \%$ & 0.077 & 0.826 & 0.765 & 0.886 & 0.315 & 0.289 & 0.342 \\
\hline $40 \%$ & 0.086 & 0.732 & 0.658 & 0.799 & 0.416 & 0.389 & 0.443 \\
\hline $50 \%$ & 0.097 & 0.664 & 0.584 & 0.738 & 0.520 & 0.493 & 0.546 \\
\hline $60 \%$ & 0.111 & 0.537 & 0.456 & 0.617 & 0.616 & 0.589 & 0.644 \\
\hline $70 \%$ & 0.123 & 0.450 & 0.369 & 0.530 & 0.717 & 0.692 & 0.743 \\
\hline $80 \%$ & 0.141 & 0.342 & 0.269 & 0.423 & 0.817 & 0.796 & 0.840 \\
\hline $90 \%$ & 0.176 & 0.208 & 0.148 & 0.282 & 0.913 & 0.898 & 0.929 \\
\hline
\end{tabular}

Age and disease history (diabetes, hypertension, acute myocardial infarction, and stroke) at baseline were included in the LR model. Confidence intervals were calculated using a R package pROC with 2000 stratified bootstrap replicates.

$\mathrm{Cl}$, confidence interval; LR, logistic regression.

Supplementary Table 6. Spearman correlations of CD40 and CD40L (A.U., CPIP cohort) measured in plaque homogenate versus plaque elements

\begin{tabular}{|c|c|c|c|c|c|c|}
\hline \multirow{2}{*}{ Variable } & \multicolumn{3}{|c|}{ CD40 (n=198) } & \multicolumn{3}{|c|}{ CD40L $(n=198)$} \\
\hline & $r$ & $P$ & $P$, adjusted & $r$ & $P$ & $P$, adjusted \\
\hline \multicolumn{7}{|c|}{ Plaque components $(\mathrm{mg} / \mathrm{g})$} \\
\hline Collagen & 0.248 & 0.00041 & 0.002 & 0.286 & $4.7 \times 10^{-5}$ & 0.00052 \\
\hline Elastin & 0.351 & $3.8 \times 10^{-7}$ & $4.2 \times 10^{-6}$ & 0.339 & $1.3 \times 10^{-6}$ & $1.7 \times 10^{-5}$ \\
\hline MMP1 & 0.262 & 0.00028 & 0.002 & 0.054 & 0.465 & 0.465 \\
\hline MMP2 & 0.254 & 0.00041 & 0.002 & 0.076 & 0.1 & 0.41 \\
\hline MMP9 & 0.249 & 0.0040 & 0.01 & 0.121 & 0.1 & 0.41 \\
\hline MMP10 & 0.373 & $1.3 \times 10^{-7}$ & $1.7 \times 10^{-6}$ & 0.373 & 0.003 & 0.021 \\
\hline TIMP1 & 0.363 & $4.2 \times 10^{-7}$ & $4.2 \times 10^{-6}$ & 0.204 & 0.006 & 0.035 \\
\hline TIMP2 & 0.326 & $6.1 \times 10^{-6}$ & $5.5 \times 10^{-5}$ & 0.307 & $2.5 \times 10^{-5}$ & 0.0003 \\
\hline TIMP3 & 0.219 & 0.048 & 0.063 & 0.153 & 0.171 & 0.409 \\
\hline
\end{tabular}

CD40L, CD40 ligand; A.U., arbitrary unit; CPIP, Carotid Plaque Imaging Project; MMP, matrix metalloproteinase; TIMP, tissue inhibitor of metalloproteinase. 